\title{
Stress selectively reduces sugar + saccharin mixture intake but increases proportion of calories consumed as sugar by rats
}

\author{
NANCY K. DESS and SEUNG CHOE \\ Occidental College, Los Angeles, California
}

\begin{abstract}
Exposure to unsignaled, inescapable tailshock reduces intake of quinine- or saccharin-adulterated water but increases intake of dilute sucrose solution. This pattern suggests that stress may reduce consumption of quinine and saccharin by enhancing their aversive taste properties. The present study provided additional support for this interpretation: Stress reduced intake of a saccharin-adulterated solution of glucose (Experiment 1) or sucrose (Experiment 2) but not of unadulterated sugar solutions. Stress also reduced body weight and total calorie intake, and increased selection of calories in the form of sugar (Experiment 2). These findings are inconsistent with the generalized "anhedonia" concept prominent in the literature on stress-induced changes in ingestion and other motivated behaviors. The selectivity of tailshock's effects on ingestion encourages further development of an energy-regulation model of stress (the regulatory shift hypothesis), including exploration of the roles that macronutrient accessibility and sugars' stressreducing, reinforcing properties might play in the behavior of stressed rats.
\end{abstract}

Stress affects intake of flavored solutions by rats (e.g., Katz, 1982; Plaznik, Stefanski, \& Kostowski, 1989; Willner, Towell, Sampson, Sophokleous, \& Muscat, 1987). An interesting pattern of results has emerged in the helplessness paradigm, in which stress consists of exposure to a session of intermittent unsignaled, inescapable tailshock. This stressor reduces intake of quinine-adulterated water or food and saccharin-adulterated water but increases intake of dilute sucrose solution (Dess, 1992; Dess \& Chapman, 1990; Dess, Chapman, \& Minor, 1988; Dess, Minor, \& Brewer, 1989). Divergent effects of tailshock on saccharin and dilute sucrose intake are inconsistent with the reduction in intake of both solutions observed in other stress paradigms and thus with the "anhedonia" invoked as an explanation (Koob, 1989; Willner, Golembiowska, Klimek, \& Muscat, 1991).

An alternative explanation is suggested by the suppressive effect of tailshock on ingestion of saccharin- and quinine-adulterated water. While saccharin and quinine differ with respect to "sweetness" or appetitive taste properties, they both have aversive taste properties in rats and humans (Dess, 1993). Enhancement of those aversive taste properties by stress could mediate suppression of saccharin and quinine intake after tailshock. Intake of sucrose,

Experiment 2 was presented at the 1993 joint meetings of the Western and Rocky Mountain Psychological Associations in Phoenix, AZ. This research was supported by NIMH Grant MH41170 to T. R. Minor and N. K. Dess, a grant from the Pew Charitable Trusts to N. K. Dess and W. S. Agras, and the Office of the Dean of Faculty at Occidental College. Correspondence should be addressed to N. K. Dess, Department of Psychology, Occidental College, 1600 Campus Road, Los Angeles, CA 90041 . which lacks aversive taste properties, would not be suppressed. The frank increase in sucrose intake after tailshock would require some other mechanism.

The putative enhancement of saccharin's aversive taste properties was explored further in the present study. The design exploited the robust behavior patterns observed in rats given sugar solutions of different concentrations, with or without saccharin adulteration. Rats given two unadulterated glucose solutions of different concentrations consume more of the concentrated solution than they do of the dilute alternative, at least for a few days (Booth, Lovett, \& McSherry, 1972). Addition of saccharin to both solutions yields the opposite pattern (Smith \& Foster, 1980). Though the effect can be called preference reversal, this terminology is misleading: Saccharin adulteration has little effect on intake of concentrated glucose solution but doubles intake of dilute glucose solution, regardless of whether the alternative to the glucose + saccharin mixture is water or another glucose solution. Thus, the apparent effect of saccharin on preference derives from modulation of dilute sugar consumption by saccharin, not from redistribution of a fixed amount of drinking between the two solutions. Avid intake of dilute glucose + saccharin solution is likely promoted by intense sweetness and inhibition of saccharin's aversive taste properties by the sugar (Smith, Foster, \& Bartoshuk, 1982; Smith, Williams, \& Jue, 1976), and is permitted by minimal postingestive (caloric) inhibition.

In the study described below, rats either were exposed to tailshock or remained in their home cages, then received a dilute and a concentrated solution of glucose (Experiment 1) or sucrose (Experiment 2). For half of the rats in each condition, the dilute solution was adulterated with 
saccharin. This design allowed assessment of the effects of stress on consumption of caloric alternatives as a function of sugar concentration and saccharin adulteration.

\section{EXPERIMENT 1}

Experiment 1 examined the effect of stress on consumption of glucose solutions of two concentrations when the more dilute solution was plain or adulterated with saccharin. Stress-induced anhedonia should reduce intake of the weak sugar solution (Muscat, Towell, \& Willner, 1988; Willner et al., 1991; Willner et al., 1987)-a prediction tested and rejected once before in the helplessness paradigm with a water/dilute-sucrose choice task (Dess, 1992). By a recent elaboration of the anhedonia concept, stress-induced anhedonia should not reduce intake of very sweet solutions, such as sugar + saccharin or concentrated sugar solution, due to saturation of "reward" functions at high sweetness values (discussed in Willner et al., 1991). Experiment 1 provides another test of the former prediction and an original test of the latter.

The idea that tailshock enhances aversive taste properties generates quite different predictions. According to this view, tailshock should selectively reduce intake of saccharin-adulterated solutions. Intake of unadulterated sugar solutions should not be reduced, and may be increased (Dess, 1992).

\section{Method}

Subjects. Thirty-nine naive adult male Holtzman rats that were the laboratory-reared offspring of pregnant rats from HSD Inc. (Indianapolis, IN) were used. At least two weeks prior to the experiment, they were individually housed on a 12:12-h light:dark cycle with light onset at 8:00 a.m. Treatments and measurements took place in the light phase. Purina 5001 chow and tap water were available ad lib prior to and throughout the experiment.

Apparatus. All drinking tests took place in the home cages. Flavored solutions were made daily with tap water. Chemicals used were sodium saccharin and quinine hydrochloride (Sigma Chemical Co., St. Louis, MO), and glucose and sucrose (ICN Biochemicals, Costa Mesa, CA). During taste pretesting, $0.1 \% \mathrm{wt} / \mathrm{vol}$ saccharin, $2 \% \mathrm{wt} / \mathrm{vol}$ sucrose, and $0.0002 \% \mathrm{wt} / \mathrm{vol}$ quinine solutions were used. During post-stress testing, three solutions were used: $24 \% \mathrm{wt} / \mathrm{vol}$ glucose, plain $2 \% \mathrm{wt} / \mathrm{vol}$ glucose, and $2 \% \mathrm{wt} / \mathrm{vol} \mathrm{glu}-$ cose adulterated with $0.1 \%$ saccharin (saccharin mixture).

The stress session was administered in individual acrylic chambers $(14 \times 11 \times 17 \mathrm{~cm})$. A rat's tail extended through the back of the chamber and was taped to a Plexiglas rod. Two electrodes coated with electrode paste were taped to the tail $1.5 \mathrm{~cm}$ apart. Shock was delivered via the electrodes from a constant-current shock generator (Lafayette Instrument Model 82400, Lafayette, IN). Shock delivery was computer-controlled. The chambers were located in individual white sound-attenuating modules, each illuminated by a bulb in the center of the front wall. Ventilation fans behind the front wall provided masking white noise.

Procedure. The experiment consisted of four phases:

Taste pretest. A pretest one week prior to the experiment consisted of a 48-h water baseline period followed by serial access to saccharin $(48 \mathrm{~h})$, quinine $(24 \mathrm{~h})$, and sucrose $(24 \mathrm{~h})$ solution, always with a tap-water alternative. The pretest served several purposes. It was used to attenuate so-called neophobia and to match groups on saccharin intake. The pretest also allowed identification of low-saccharin rats-defined as rats whose saccharin intake on both pretest days was below water baseline--for exclusion from the analyses below. Low-saccharin rats were used in a selectivebreeding study of saccharin intake and its relation to emotionality, which thus far suggests that low saccharin intake predicts low intake of saccharin-adulterated sugar solution and perhaps deviant responses to stress (Dess \& Minor, 1994). All pretest data from Experiment 1 are included in that report as laboratory-reared random-bred controls for taste tests on selectively bred rats.

Prestress baselines. Water intake $( \pm 0.5 \mathrm{ml})$ was measured for $48 \mathrm{~h}$ preceding the stress session to establish a new water baseline. Just prior to the stress session, body weights were measured to the nearest $0.5 \mathrm{~g}$.

Stress session. Rats were assigned to one of four groups, balanced for baseline water intake and body weight. Two groups were stressed. The stress session began at approximately 9:00 a.m. Stress consisted of exposure to $1005-\mathrm{sec}, 1.0-\mathrm{mA}$ tailshocks on a variabletime 60-sec (range: $20-230 \mathrm{sec}$ ) schedule. Nonstressed groups remained in the home cages.

Testing. Immediately after stressed rats were returned to their home cages, all rats were given a preweighed bottle of $24 \%$ glucose solution and a preweighed bottle of $2 \%$ glucose solution. The $2 \%$ solution was unadulterated for one nonstressed group and one stressed group (No Stress-Plain and Stress-Plain, each $n=10$ ) and was adulterated with saccharin for the other two groups (No Stress-Mixture, $n=9$, and Stress-Mixture, $n=10$ ).

Bottles were weighed and solutions were replaced with fresh ones the next day between 10:00 a.m. and noon. Testing concluded $48 \mathrm{~h}$ after the stress session with a final weighing of the bottles. Fluid intake in $\mathrm{ml}$ was calculated based on the weight $(\mathrm{ml} / \mathrm{g})$ of the various solutions.

Statistics. Analysis began with a multiway analysis of variance (ANOVA). Significant interactions were analyzed with follow-up ANOVAs in which one or more variables were held constant. $F$ is reported below only for effects significant at $\alpha=.05$. All data in the text and figures are given as means and standard errors.

\section{Results and Discussion}

The groups did not differ on any preexperimental measure. No low-saccharin rats were identified in the pretest. On average, saccharin intake during the pretest exceeded water baseline by $54.5 \pm 5.4 \mathrm{ml}$. Prestress body weight averaged $556.9 \pm 34.7 \mathrm{~g}$, and daily water intake averaged $61.2 \pm 1.6 \mathrm{ml}$.

The amount of each solution consumed on the two test days is shown in Figure 1. When $24 \%$ glucose and plain

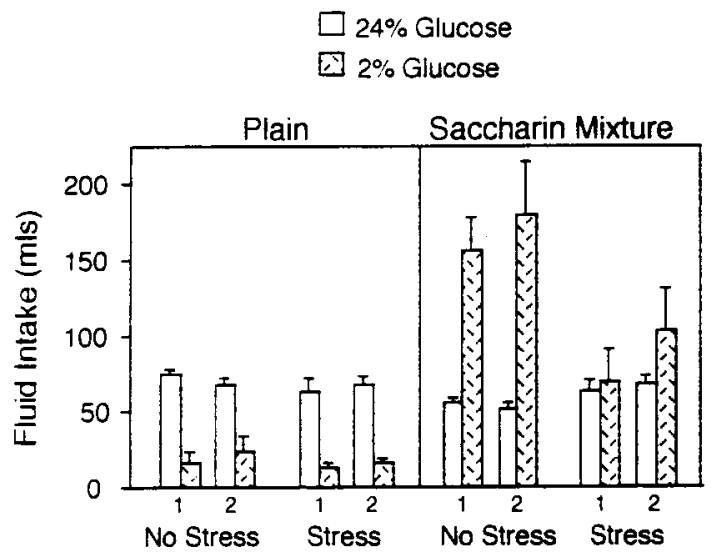

Figure 1. Consumption of glucose solutions $(\mathrm{ml})$ by nonstressed and stressed rats in two 24-h tests (Experiment 1). All rats received $24 \%$ glucose solution and $2 \%$ glucose solution; the latter was adulterated with saccharin $(0.1 \%)$ for the Mixture groups. 
$2 \%$ glucose were available, rats consumed more of the concentrated solution than they did of the dilute solution whether or not they had been stressed; absolute amount consumed of each solution also was unaffected by stress. When $24 \%$ glucose and the saccharin mixture were available, nonstressed rats consumed more of the mixture than they did of the concentrated solution. Stressed rats, on the other hand, consumed about equal amounts of the two solutions. These patterns changed little from the first to the second test day, except for a general tendency for dilute solution intake to increase from Day 1 to Day 2 .

The data were analyzed with a four-way ANOVA using stress condition (no stress vs. stress) and $2 \%$ glucose flavor (plain vs. mixture) as between-groups variables and concentration ( $24 \%$ vs. $2 \%$ ) and test day as repeated measures. This ANOVA yielded main effects of stress, flavor, and day $[F(1,35)=4.73,29.37$, and 5.06, respectively $]$ and interactions of concentration with flavor, stress, and day $[F \mathrm{~s}(1,35)=40.79,6.28$, and 5.71, respectively]. A stress $\times$ flavor $\times$ concentration interaction $[F(1,35)=$ 6.18] was analyzed further with separate stress $\times$ concentration $\times$ day ANOVAs on the Plain groups' data and on the Mixture groups' data. In the Plain condition, no effects involving stress were significant. In the Mixture condition, the stress $\times$ concentration interaction was significant $[F(1,17)=6.80]$ : A concentration $\times$ day ANOVA on the No Stress-Mixture group's data yielded only a concentration effect $[F(1,8)=18.98]$, reflecting the high intake of saccharin mixture relative to $24 \%$ glucose; the same ANOVA on the Stress-Mixture group's data yielded no concentration or day effects.

As expected, unstressed rats consumed more concentrated than dilute plain glucose solution, and saccharin adulteration dramatically increased their intake of the dilute solution. Stress had no effect on relative or absolute intake of the two plain glucose solutions but selectively reduced intake of saccharin-adulterated glucose solution. This pattern of results is at odds with the anhedonia concept and, considered together with similarly disconfirming evidence from an earlier study (Dess, 1992), argues strongly against anhedonia as a mediator of flavor intake after unsignaled, inescapable tailshock. These and prior findings consistently support an interpretation in terms of enhancement of saccharin's aversive taste properties by stress.

\section{EXPERIMENT 2}

Experiment 2 was a replication of Experiment 1 with a few important changes. Equicaloric sucrose solutions replaced glucose solutions. In many respects, rats react similarly to sucrose and glucose availability. For example, intake of a solution made with either type of sugar is increased synergistically by saccharin adulteration (Sclafani, Einberg, \& Nissenbaum, 1987), and rats consume approximately half of their calories as sugar when chow and either sucrose or glucose solution are available (Castonguay, Hirsch, \& Collier, 1981). In the present study, though, results for sucrose could differ from those for glucose (Experiment 1). Sucrose and glucose differ chemically and physiologically (Grill, Berridge, \& Ganster, 1984; Sclafani, 1987). To the extent that cephalic phase or post-ingestional mechanisms mediate the effects of stress observed in Experiment 1, sucrose could yield a different outcome. Furthermore, sucrose is sweeter than glucose and therefore may more effectively inhibit saccharin's aversive taste properties (Lawless, 1979; Smith, Foster, \& Bartoshuk, 1982). Stronger inhibition might offset the putative enhancement of those properties by stress, closing the gap between stressed and nonstressed rats' sugar + saccharin intake.

Experiment 2 also was designed to provide a fuller picture of ingestive processes after tailshock. Daily chow consumption was measured, which permitted assessment of total caloric intake and the apportionment of caloric intake between sugar and chow. In addition, rats were weighed daily. In a previous study, stress reduced body weight when saccharin solution was available to ad-lib chow-fed rats, but not when a dilute sucrose solution was available to them (Dess, 1992). Whether access to calorically dense or saccharin-adulterated sugar solution would prevent weight loss as well was of interest. Body weight measurement also allowed calculation of a crude index of the disposition of ingested calories after stress. Tailshock can cause weight loss even when food intake is spared (Dess et al., 1989, Experiment 3). Dissociation between changes in body weight and food consumption indicates that weight loss is not entirely a by-product of reduced food intake (or vice versa; Friedman, 1992). The congruence between body weight and food intake when several caloric media are available after stress was examined in Experiment 2.

Finally, Experiment 2 included repetition of the No Stress-Mixture condition from Experiment 1-that is, nonstressed rats given $24 \%$ glucose and saccharinadulterated $2 \%$ glucose. This group was included for two reasons. First, it allowed direct comparison of calorie intake and selection in the No Stress-Mixture condition using sucrose versus using glucose. Similarity of No Stress-Mixture (sucrose) and No Stress-Mixture (glucose) groups in Experiment 2 would strengthen inferences about Experiment 1 from Experiment 2. Second, the rats used in Experiment 2 were reared by the supplier, not in the laboratory. Comparing the No Stress-Mixture (glucose) groups of Experiments 1 and 2 provided a measure of the consistency of glucose and glucose + saccharin intake across rearing conditions.

\section{Method}

Except as stated otherwise, the method for Experiment 2 was identical to that of Experiment 1.

Subjects and Materials. Fifty-eight naive male Holtzman rats (HSD Inc., Indianapolis, IN) began the study. Five low-saccharin rats were identified in the pretest and were excluded from analyses of the poststress data. Daily saccharin intake during the pretest among these 5 rats averaged $42.1 \pm 7.3 \mathrm{ml}$ below water baseline, compared with an average of $40.8 \pm 4.4 \mathrm{ml}$ above baseline for the other 53 rats. 
During poststress testing, five solutions were used: $24 \% \mathrm{wt} / \mathrm{vol}$ sucrose solution, $24 \% \mathrm{wt} / \mathrm{vol}$ glucose solution, plain $2 \% \mathrm{wt} / \mathrm{vol}$ sucrose solution, and saccharin-adulterated $2 \%$ sucrose and $2 \%$ glucose solutions.

Procedure. After the last baseline measurement, rats were assigned to one of five groups, balanced for prestress saccharin, water, and chow intake and for body weight. The group treatments constituted a $2 \times 2+1$ design, with stress (shock vs. no shock) and saccharin adulteration (plain vs. mixture) as the variables in the factorial part of the design. Rats in those four conditions were tested with sucrose solutions, whereas the fifth group was tested with glucose.

Two of the groups were stressed. After the stress session, all groups were given a $24 \%$ sugar solution and a $2 \%$ sugar solution for three days. One nonstressed group and one stressed group were given $24 \%$ sucrose and unadulterated $2 \%$ sucrose solutions (No Stress-Plain and Stress-Plain; each $n=11$ ). Another nonstressed group and another stressed group were given $24 \%$ sucrose and saccharin-adulterated $2 \%$ sucrose solutions (No Stress-Mixture and Stress-Mixture, each $n=11)$. The fifth group $(n=9)$ was given $24 \%$ glucose and saccharin-adulterated $2 \%$ glucose solutions. Fluid intake, chow intake, and body weight were measured daily for three days. Calorie consumption was calculated from the weight $(\mathrm{ml} / \mathrm{g}$ or $\mathrm{g}$ ) and caloric density ( $\mathrm{kcal} / \mathrm{ml} \mathrm{or} \mathrm{kcal} / \mathrm{g}$ ) of the various solutions and of chow.

\section{Results and Discussion}

Baselines. The groups did not differ on any prestress measure. Prestress means were $475.0 \pm 3.8 \mathrm{~g}$ for body weight, $52.2 \pm 1.6 \mathrm{ml}$ for daily water intake, and 101.0 $\pm 1.5 \mathrm{kcal}$ for chow intake.

Fluid intake. Fluid intake during testing for each of the four sucrose groups is shown in Figure 2 . When $24 \%$ and plain $2 \%$ sucrose were available, rats consumed more of the concentrated solution than they did of the dilute solution, regardless of stress; absolute amount consumed of each solution differed little between stressed and nonstressed Plain groups. When $24 \%$ sucrose and saccharin mixture were available, nonstressed rats consumed more of the mixture than they did of the concentrated solution. Stressed rats, on the other hand, initially consumed about equal amounts of the two solutions, then increased their

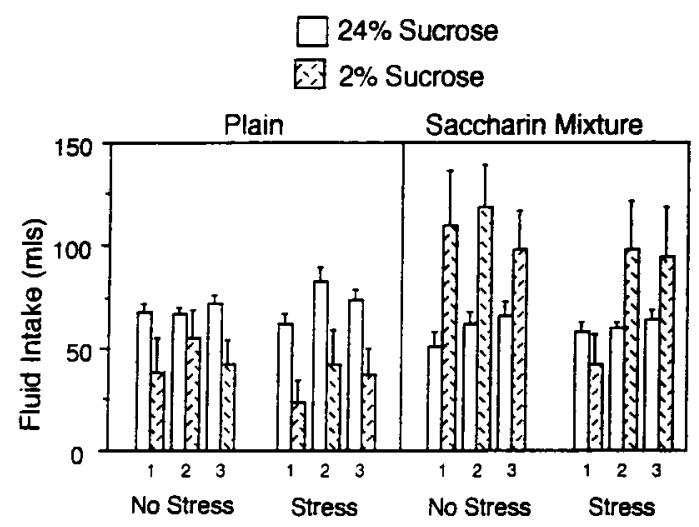

Figure 2. Consumption of sucrose solutions ( $\mathrm{ml}$ ) by nonstressed and stressed rats in three 24-h tests (Experiment 2). All rats received $\mathbf{2 4 \%}$ sucrose solution and $2 \%$ sucrose solution; the latter was adulterated with saccharin $(0.1 \%)$ for the Mixture groups.

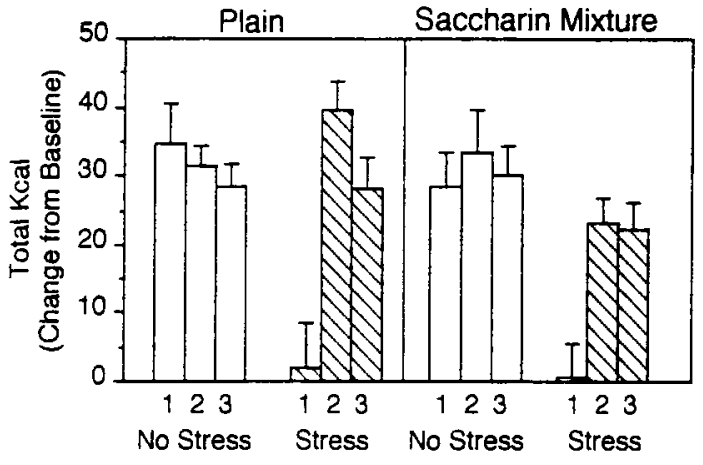

Figure 3. Total calorie intake (sucrose kcal + chow kcal), as change from prestress calorie baseline (chow kcal), on Test Days 1-3 (Experiment 2).

intake of saccharin mixture markedly. Plain and Mixture groups showed some tendency for intake of the dilute solution to increase from Day 1 to Day 2. Though variability was somewhat greater than it was in Experiment 1, the pattern of results is basically the same: Stress selectively reduced intake of saccharin-adulterated sugar solution. In contrast to Experiment 1, however, intake of the saccharin mixture recovered significantly within $48 \mathrm{~h}$.

A stress $\times$ flavor $x$ concentration $\times$ day ANOVA on these data yielded main effects of flavor $[F(1,40)=7.65]$ and day $[F(2,80)=11.71]$, as well as of stress $\times$ day $[F(2,80)=3.85]$ and flavor $\times$ concentration $[F(1,40)=$ 12.18] interactions. A stress $\times$ flavor $\times$ concentration $\times$ day interaction $[F(2,80)=3.73]$ was further examined in separate three-way ANOVAs for the Plain and Mixture conditions. In the Plain condition, no effects involving stress were significant. In the Mixture condition, the stress $\times$ concentration $\times$ day interaction was significant $[F(2,40)=4.43]$. A concentration $\times$ day ANOVA on the No Stress-Mixture group's data yielded a main effect of concentration $[F(1,10)=5.15]$. The same ANOVA on the Stress-Mixture group's data yielded a concentration $X$ day interaction $[F(2,20)=6.94]$. Follow-up ANOVAs showed that among stressed rats, intake of $24 \%$ sucrose did not change over test days whereas intake of saccharin mixture did $[F(2,20)=9.14]$; specifically, saccharin mixture intake increased from Day 1 to Day $2[t(20)=$ 3.79], but not from Day 2 to Day 3 .

Total calorie intake. Total calorie intake is shown as change from baseline in Figure 3. Access to the sugar solutions increased caloric intake from baseline levels in nonstressed but not in stressed rats. The reduction of calorie intake by stress was comparable in the Plain and Mixture conditions. Recovery from stress was fast, particularly when plain $2 \%$ sucrose was available.

A stress $\times$ flavor $\times$ day ANOVA yielded main effects of stress $[F(1,40)=11.51]$ and day $[F(2,80)=15.31]$ and a stress $\times$ day interaction $[F(2,80)=15.75]$. No effect involving flavor was significant. A stress $\times$ flavor ANOVA for Day 1 showed only a main effect of stress $[F(1,40)=28.80]$. On Day 2, however, the stress $X$ 
flavor interaction was significant $[F(1,40)=4.06]$ : Saccharin adulteration reduced calorie intake among stressed rats $[F(1,20)=8.43]$, but had no effect in the nonstressed condition. No stress or flavor effects remained on Day 3.

Calorie selection. The percentage of total daily calories consumed as sugar [Total sucrose $\mathrm{kcal} /$ Total $\mathrm{kcal}$ ] is shown in Figure 4. Stress increased the selection of calories in the form of sucrose, regardless of whether the dilute sucrose solution was plain or adulterated with saccharin. The difference between stressed and nonstressed groups diminished over test days, as nonstressed rats increasingly selected calories from sucrose rather than from chow. A stress $\times$ flavor $\times$ day ANOVA yielded a main effect of stress $[F(1,40)=5.67]$ and a stress $\times$ day interaction $[F(2,80)=10.25]$. Separate stress $\times$ flavor ANOVAs on each test day showed only an effect of stress on Day $1[F(1,40)=12.69]$. No effect involving flavor was significant.

The percentage of calories taken as sugar by stressed rats was relatively stable across test days, suggesting that the recovery in total calorie intake after stress derived equally from sucrose and chow intake. Analysis of the changes in sucrose and chow intake from Day 1 to Day 2 among stressed rats showed that both sucrose and chow intake increased from Day 1 to Day $2(+13.8 \pm 5.4 \mathrm{kcal}$ and $+16.5 \pm 2.1 \mathrm{kcal}$, respectively). Thus, increases in sucrose and chow intake contributed about equally to the recovery in total food consumption after stress.

These results help reconcile the present and earlier findings. While absolute intake of unadulterated sugar solutions was spared after stress in Experiments 1 and 2, it did not increase as it had been observed to do before (Dess, 1992). With sugar intake expressed as selection among caloric alternatives in Experiment 2, however, stress did increase sugar intake. Perhaps absolute sugar intake did not increase here due to the use of concentrated, calorically dense sugar solutions, which strongly regulate further ingestion.

Body weight. Body weight during testing is shown as change from baseline in Figure 5. Stress reduced body weight relative to the no-stress condition and relative to

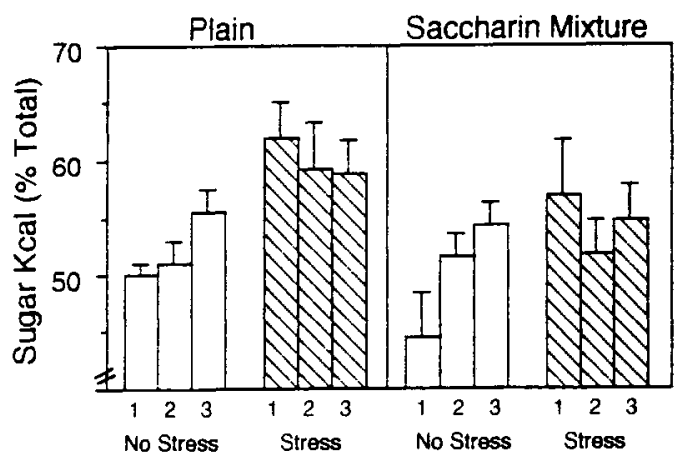

Figure 4. Percentage of total daily calories consumed as sucrose (total sucrose kcal/total kcal), on Test Days 1-3 (Experiment 2). Note that the $y$ axis begins just above $40 \%$ rather than at $0 \%$.

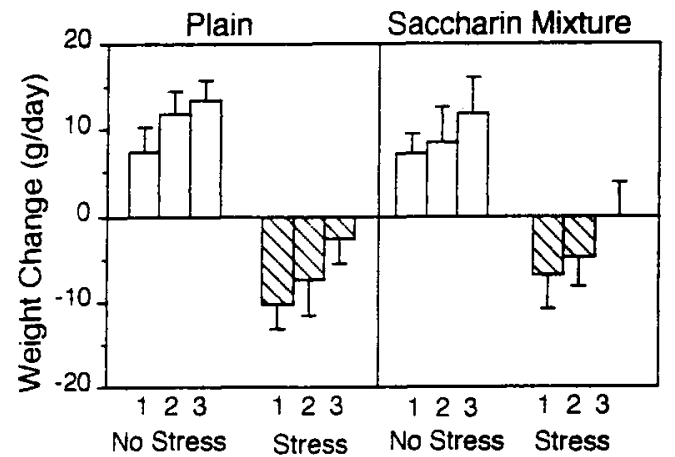

Figure 5. Body weight (g), as change from prestress body weight baseline, on Test Days 1-3 (Experiment 2).

the prestress baseline. Saccharin adulteration of the $2 \%$ sucrose solution had no effect on body weight. A stress $x$ flavor $X$ day ANOVA yielded only main effects of stress $[F(1,40)=25.09]$ and day $[F(2,80)=12.47]$.

Weight loss after tailshock has been observed under many conditions (Dess, 1992; Dess et al., 1989; Dess, Raizer, Chapman, \& Garcia, 1988; Weiss, 1968) but, interestingly, not during access to dilute sucrose solution (Dess, 1992). The reasons for weight loss in Experiment 2 among shocked rats, who also had access to sugar, are unclear. Their relatively high intake of calories as simple carbohydrate solutions may have played a role, for example, via potent postingestive inhibition or by reducing chow intake and calorie absorption in the gut (Rivest, Deshaies, \& Richard, 1989). The mechanism(s) must interact with stress because concentrated sugar solutions normally do not reduce body weight (Castonguay et al., 1981). Relevant data are provided in the following analyses.

Fuel "partitioning." These data provide several examples of dissociation between the effects of stress on caloric intake and body weight. On Day 1 , stressed rats consumed as many calories as they had done prior to stress (see Figure 3) but nevertheless lost weight. Furthermore, stressed rats gained little weight between Days 1 and 2 $(2.4 \pm 1.7 \mathrm{~g})$ despite dramatic recovery in total calorie intake. A comparable increase in calorie intake from baseline to Day 1 by nonstressed rats produced significantly greater weight gain $[7.4 \pm 1.6 \mathrm{~g}, t(40)=2.11]$.

A crude measure of fuel partitioning was calculated by dividing each rat's weight change by total calorie intake for each test day. This measure reflects gross body weight yield per ingested kcal. If group differences in body weight are linked solely to differences in total calorie intake, effects of stress should disappear with this derived measure. If, on the other hand, stress alters the partitioning of ingested food into storage (vs. utilization and waste), stress effects should remain.

Weight gain per kcal was initially reduced by stress, then recovered over test days (Figure 6). Saccharin adulteration was irrelevant to these effects. A stress $\times$ flavor $\times$ day ANOVA showed main effects of stress $[F(1,40)=7.78]$ 


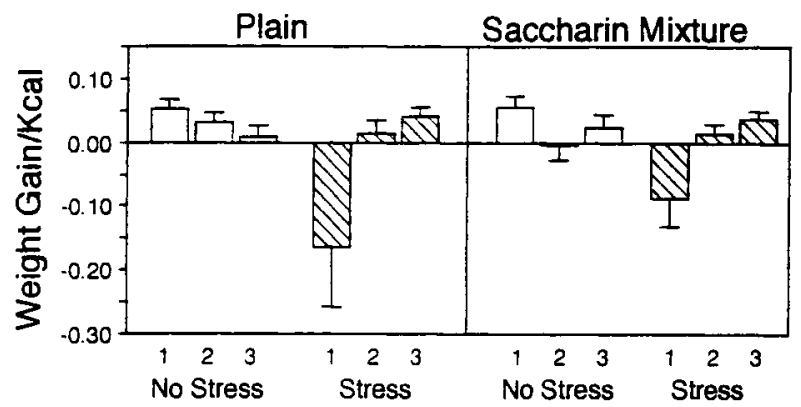

Figure 6. Fuel partitioning on Test Days 1-3 in Experiment 2: This crude measure of how much ingested food was stored rather than lost or utilized was derived by dividing daily change in body weight by total calories ingested over the same 24-h period, yielding a "g/kcal" score.

and day $[F(2,80)=3.53]$ and a stress $\times$ day interaction $[F(2,80)=9.94]$. Follow-up flavor $\times$ day ANOVAs showed a main effect of day for stressed rats $[F(2,40)=$ 7.74] and no significant effect of flavor or day for nonstressed rats. In addition to affecting how many calories are consumed and from what source, tailshock appears to alter the fate of ingested calories.

Comparison of sucrose and glucose. The two nonstressed groups given $24 \%$ and saccharin-adulterated $2 \%$ solutions of sucrose or glucose were compared on each of the measures analyzed above. In no case did the groups differ significantly. Thus, conclusions drawn with sucrose in Experiment 2 may generalize fairly well to glucose (Experiment 1).

\section{GENERAL DISCUSSION}

Inescapable, unsignaled tailshock selectively reduces intake of saccharin-adulterated sugar solution. The sugar + saccharin mixtures might be "too sweet" for stressed rats, but some results work against this interpretation. For nonstressed and stressed rats alike, intake of the more concentrated (and sweeter) of two plain sugar solutions exceeds intake of the weaker one whether the solutions are $1 \%$ and $2 \%$ sucrose (Dess, 1992), $2 \%$ and $24 \%$ glucose (Experiment 1), or 2\% and 24\% sucrose (Experiment 2). Moreover, intake of a concentrated glucose solution and intake of an equicaloric but much sweeter sucrose solution are both unaffected by stress. Thus, stressed rats discriminate normally among sugar concentrations and show little sign of being unresponsive to sweetness over a broad range of sweetness values.

These data support the idea that stress increases saccharin's aversive taste properties. Inhibition of saccharin's aversive taste by sucrose or glucose may potentiate intake of sugar + saccharin mixtures (Smith et al., 1982) but may do so less completely after stress. This interpretation provides a parsimonious account of the present result and of the finding that shock reduces intake of quinineadulterated water or food and saccharin-adulterated water but not of water or dilute sucrose solution (Dess, 1992;
Dess \& Chapman, 1990; Dess et al., 1988; Dess et al., 1989).

Recovery of calorie intake also was modulated by saccharin adulteration: Total calorie intake recovered more slowly to the level of nonstressed controls when saccharinadulterated sucrose solution was available. In contrast, saccharin adulteration was irrelevant to the initial reduction by stress of total calorie intake, weight gain, and fuel storage and to the increase in the proportion of calories consumed as sugar. Thus, these effects were not simple by-products of the selective reduction in fluid intake due to saccharin adulteration.

While the impact of stress on separate, specific ingestive processes warrants further investigation, development of frameworks that integrate diverse behavioral and biological effects related to ingestion also is worthwhile. The regulatory shift hypothesis (Dess, 1991) places stressinduced changes in ingestive behavior in the broader context of energy regulation. Under many conditions, such as food deprivation or cold temperature, energy stored as glycogen and fat is mobilized, with several interrelated consequences. Chief among these are changes in body weight, food intake, and the relative acceptability of tastes (Friedman, 1992; Jacobs \& Sharma, 1969). Stress also mobilizes energy stores (Atkinson \& Milsum, 1983); thus, tailshock-induced weight loss, anorexia, and "finickiness" may reflect an organized shift in energy regulation. This shift allows the animal to meet its metabolic needs efficiently and at minimal risk of further stress (see also Dess \& VanderWeele, in press; Helmstetter \& Fanselow, 1993).

The increased selection of calories from sugar solution after stress is a new result with many possible mechanisms within an energy-regulation framework. Relative to chow, sugar may have tasted better to stressed rats. Sugar solution is more easily consumed and absorbed than is chow, which could be more critical to stressed rats. A shift in the optimal level of protein intake or the carbohydrate/ protein ratio (Castonguay et al., 1981) may play a role in disproportionate sugar intake after stress. Finally, sugars appear to have stress-reducing and other potentially reinforcing properties (Ahlers, Shurtleff, Schrot, Thomas, \& Paul-Emile, 1993; Blass, Shide, \& Weller, 1989; Gold, 1991), which could enhance intake of glucose or sucrose solutions. Additional research is needed to determine the mechanisms of stress-induced shifts in dietary selection.

Fuel storage was measured crudely in this study, and its apparent reduction by stress could have many causes, not all of which are directly related to energy regulation. It could not have been due to fluid intake per se: Stress affected fluid intake only when the $2 \%$ sugar solution was adulterated with saccharin but reduced weight gain $/ \mathrm{kcal}$ to the same degree regardless of saccharin adulteration. Altered fluid regulation (i.e., retention and metabolic use; Biglieri, Arteaga, \& Kater, 1989), however, could have contributed to the apparent reduction in weight gain $/ \mathrm{kcal}$ by deflating the numerator of this index. There is, nonetheless, good reason to suspect involvement of energy regu- 
lation. For example, intracerebral infusion of corticotropinreleasing factor, a major stress neurohormone, reduces food absorption in the gut and thus weight gain per ingested $\mathrm{kcal}$ in male rats (Rivest et al., 1989). Stress also stimulates lipolysis (Atkinson \& Milsum, 1983), further reducing fat storage, food intake, and weight gain $/ \mathrm{kcal}$ (Friedman, Tordoff, \& Ramirez, 1986). Reduced storage of fuel as fat and glycogen via these and related behavioral and physiological processes may reflect the heightened importance of meeting the animal's immediate metabolic needs and the diminished importance of storing fuel for future use during periods of stress.

Integration of stress effects with energy regulation suggests a way of reconciling disparities in the ingestion and stress literatures. For example, food deprivation, tailshock, and chronic exposure to varied stressors all mobilize energy stores but have different effects on taste: Food deprivation reduces quinine intake but increases saccharin and sugar intake (Gilbert \& Sherman, 1970; Jacobs \& Sharma, 1969; Mook \& Cseh, 1981; Smith \& Duffy, 1957; Valenstein, 1967); restraint (Plaznik et al., 1989) or tailshock reduces quinine and saccharin intake but increases sugar intake (Dess, 1992; Dess \& Chapman, 1990; Dess et al., 1988; Dess et al., 1989; and present study); chronic stress reduces saccharin and sucrose intake (Katz, 1982; Muscat et al., 1988; Willner et al., 1991; Willner et al., 1987). These apparent discrepancies, particularly with respect to saccharin, are less surprising given that the impact of metabolic challenges depends critically on their severity and suddenness (see Stricker \& Zigmond, 1976). Perhaps mild, slow-onset challenges enhance saccharin's appetitive properties more than they enhance its aversive ones, yielding a "sugar-like" response, whereas more severe, sudden challenges differentially enhance saccharin's aversive properties, yielding a "quinine-like" response. Chronic stress may lead to a profound, long-term shift to use of stored energy which manifests as a relatively nonselective reduction in ingestive and other behaviors. (Procedural variables that may contribute to differences between effects of stress on sugar intake in the helplessness and chronic-stress paradigms are discussed in Dess, 1991.)

Applying a biobehavioral system heuristic to stressinduced changes in ingestion may lead to some questions and answers that applying an "impairment" or "disorder" perspective will not. The system approach can exploit the literature on interrelated energetic processes in the absence of experimental stress as a framework for addressing regulatory shifts set into motion by stress. Basic questions of energy regulation and its reorganization by stress then can be distinguished from questions concerning the healthfulness or desirability of stress-induced changes in rats and the human phenomena they model, which are related-but separate-issues.

\section{REFERENCES}

Ahlers, S. T., Shurtleff, D., Schrot, J., Thomas, J. R., \& PaulEMILE, F. (1993). Glucose attenuates cold-induced impairment of delayed matching-to-sample performance in rats. Psychobiology, 21, 87-92.

Atkinson, C., \& Milsum, J. H. (1983). A system model of the metabolic response to stress. Behavioral Science, 28, 268-273.

Biglieri, E. G., Arteaga, E., \& Kater, C. E. (1989). Effect of ACTH on aldosterone and other mineralocorticoid hormones. Annals of the New York Academy of Sciences, 512, 426-437.

Blass, E. M., Shide, D. J., \& Weller, A. (1989). Stress-reducing effects of ingesting milk, sugar, and fats: A developmental perspective. Annals of the New York Academy of Sciences, 575, 292-306.

Booth, D. A., Lovett, D., \& MCSherry, G. M. (1972). Postingestive modulation of the sweetness preference gradient in the rat. Journal of Comparative \& Physiological Psychology, 78, 485-512.

Castonguay, T. W., Hirsch, E., \& Collier, G. (1981). Palatability of sugar solutions and dietary selection? Physiology \& Behavior, 27, 7-12.

DESs, N. K. (1991). Ingestion and emotional health. Human Nature, 2, 235-269.

DESS, N. K. (1992). Divergent responses to saccharin and sucrose availability after stress in rats. Physiology \& Behavior, 52, 115-125.

DEss, N. K. (1993). Saccharin aversive taste in rats: Evidence and implications. Neuroscience \& Biobehavioral Reviews, 17, 359-372.

DEss, N. K., \& ChapMaN, C. D. (1990). Individual differences in taste, body weight, and depression in the "helplessness" rat model and in humans. Brain Research Bulletin, 24, 669-676.

Dess, N. K., Chapman, C. D., \& Minor, T. R. (1988). Inescapable shock increases finickiness about drinking quinine-adulterated water in rats. Learning \& Motivation, 19, 408-422.

Dess, N. K., \& MinoR, T. R. (1994). Finickiness and emotionality: Evidence for linkage from rats selectively bred for high vs. low saccharin intake. Manuscript submitted for publication.

Dess, N. K., Minor, T. R., \& Brewer, J. (1989). Suppression of feeding and body weight by inescapable shock: Modulation by quinine adulteration, stress reinstatement, and controllability. Physiology \& Behavior, 45, 975-983.

Dess, N. K., Raizer, J., Chapman, C. D., \& Garcia, J. (1988). Effects of stressors in the learned helplessness paradigm on body weight and taste aversion in rats. Physiology \& Behavior, 44, 483-490.

Dess, N. K., \& VANDERWEele, D. A. (in press). Lithium chloride and inescapable, unsignaled tailshock differentially affect meal patterns of rats. Physiology \& Behavior.

Friedman, M. I. (1992). Metabolic control of calorie intake. In M. I. Friedman, M. G. Tordoff, \& M. R. Kare (Eds.), Chemical senses: Vol. 4. Appetite \& nutrition (pp. 19-38). New York: Marcel Dekker.

Friedman, M. I., TordofF, M. G., \& Ramirez, I. (1986). Integrated metabolic control of food intake. Brain Research Bulletin, 17, 855859.

Gilbert, R. M., \& Sherman, I. P. (1970). Palatability-induced polydipsia: Saccharin, sucrose, and water intake in rats, with and without food deprivation. Psychological Reports, 27, 319-325.

GoLD, P. E. (1991). An integrated memory system: From blood to brain. In R. C. A. Frederickson, J. L. McGaugh, \& D. L. Felten (Eds.), Peripheral signaling of the brain: Role in neural-immune interactions and learning and memory (pp. 391-419). Lewiston, NY: Hogrefe \& Huber.

Grill, H. J., Berridge, K. C., \& Ganster, D. J. (1984). Oral glucose is the prime elicitor of preabsorptive insulin secretion. American Journal of Physiology, 246, R88-R95.

Helmstetter, F. J., \& Fanselow, M. S. (1993). Aversively motivated changes in meal patterns of rats in a closed economy: The effects of shock density. Animal Learning \& Behavior, 21, 168-175.

Jacobs, H., \& Sharma, K. (1969). Taste versus calories: Sensory and metabolic signals in the control of food intake. Annals of the New York Academy of Science, 157, 1084-1125.

KATZ, R. J. (1982). Animal model of depression: Pharmacological sensitivity of a hedonic deficit. Pharmacology, Biochemistry \& Behavior. 16, 965-968.

KooB, G. F. (1989). Anhedonia as an animal model of depression. In G. F. Koob, C. L. Ehlers, \& D. J. Kupfer (Eds.), Animal models of depression (pp. 162-186). Harrisonburg, VA: R. R. Donnelley. LAWLESS, H. T. (1979). Evidence for neural inhibition in bittersweet 
taste mixtures. Journal of Comparative \& Physiological Psychology, 93, 538-547.

MoOK, D., \& CSEH, C. (1981). Release of feeding by the sweet taste in rats: The influence of body weight. Appetite, 2, 15-34.

Muscat, R., Towell, A., \& Willner, P. (1988). Changes in dopamine autoreceptor sensitivity in an animal model of depression. Psychopharmacology, 94, 545-550.

Plaznik, A., Stefanski, R., \& Kostowski, W. (1989). Restraint stressinduced changes in saccharin preference: The effect of antidepressive treatment and diazepam. Pharmacology, Biochemistry \& Behavior, 33, 755-759.

Rivest, S., Deshaies, Y., \& Richard, D. (1989). Effects of corticotropin-releasing factor on energy balance in rats are sex dependent. American Journal of Physiology, 257, R1417-RI422.

SCLAFANI, A. (1987). Carbohydrate taste, appetite, and obesity: An overview. Neuroscience \& Biobehavioral Reviews, 22, 131-153.

Sclafani, A., Einberg, L. T., \& Nissenbaum, J. W. (1987). Influence of saccharin on Polycose, sucrose, and glucose intake and preference in rats. Neuroscience \& Biobehavioral Reviews, 11, 223-229.

Smith, J. C., \& Foster, D. F. (1980). Some determinants of intake of glucose + saccharin solutions. Physiology \& Behavior, 25, 127-133.

Smith, J. C., Foster, D. F., \& Bartoshuk, L. M. (1982). The synergistic properties of pairs of sweeteners. In L. M. Barker (Ed.), The psychobiology of human food selection (pp. 123-138). Westport, CT: Avi Publishing.
Smith, J. C., Willuams, D. P., \& Jue, S. S. (1976). Rapid oral mixing of glucose and saccharin by rats. Science, 191, 304-305.

SMITH, M. \& DUFFY, M. (1957). Consumption of sucrose and saccharine by hungry and satiated rats. Journal of Comparative \& Physiological Psychology, 50, 65-69.

Stricker, E. M., \& Zigmond, M. J. (1976). Brain catacholamines and the lateral hypothalamic syndrome. In D. Novin, W. Wyrwicka, \& G. Bray (Eds.), Hunger: Basic mechanisms and clinical implications (pp. 19-32). New York: Raven Press.

ValENSTEIN, E. S. (1967). Selection of nutritive and nonnutritive solutions under different conditions of need. Journal of Comparative \& Physiological Psychology, 63, 429-433.

WEISs, J. M. (1968). Effects of coping responses on stress. Journal of Comparative \& Physiological Psychology, 65, 251-260.

Willner, P., Golembiowska, K., Klimek, V., \& Muscat, R. (1991). Changes in mesolimbic dopamine may explain stress-induced anhedonia. Psychobiology, 19, 79-84.

Willner, P., Towell, A., Sampson, D., Sophokleous, S., \& MusCAT, R. (1987). Reduction of sucrose preference by chronic unpredictable mild stress, and its restoration by a tricyclic antidepressant. Psychopharmacology, 93, 358-364.

(Manuscript received August 2, 1993; revision accepted for publication October 19, 1993.) 